\title{
Ciclo de Vida de Tecnologías y Curvas en S Aplicadas en Subproductos de la Agroindustria Piscícola
}

\author{
Jhon W. Zartha ${ }^{(1) \star}$, Darwin F. Zuluaga ${ }^{(1)}$, Juan C. Palacio ${ }^{(1)}$ y Juan M. Montes ${ }^{(2)}$ \\ (1) Universidad Pontificia Bolivariana, Facultad de Ingeniería Agroindustrial, Circular 1a. 70-01 Medellín, \\ Colombia. (e-mail: jhon.zartha@upb.edu.co; darwin@universitarios.com; juan.palacio@upb.edu.co) \\ (2) Universidad de Medellín, Facultad de Ciencias Económicas y Administrativas, Carrera $87 N^{\circ} 30-65$ \\ Medellín - Colombia. (e-mail: jmontes@udem.edu.co)
}

* Autor a quien debe ser dirigida la correspondencia.

Recibido Jul. 28, 2016; Aceptado Sep. 22, 2016; Versión final Nov. 18, 2016, Publicado Abr. 2017

\begin{abstract}
Resumen
Se analiza el ciclo de vida de tecnologías disponibles a través del método de las curvas en $S$ en tres tecnologías: proteína hidrolizada, alimentación animal y probióticos, relacionadas con subproductos de la industria piscícola. Se construyeron estrategias de búsqueda para artículos y patentes en bases de datos especializadas tales como Scopus y Free Patents On-line. Para el análisis de la información se utilizó las curvas en $\mathrm{S}$ a través del cálculo del punto de inflexión. Esto permite determinar el estado de la tecnología dentro de su ciclo de vida y ayudar a la toma de decisiones frente al momento adecuado para ejercer mecanismos de propiedad intelectual y derecho tecnológico y sugerir estrategias de monitoreo e inversión. Entre los resultados más importantes se observa que considerando artículos publicados, se encuentra que dos de las tecnologías analizadas, proteína hidrolizada y probióticos no han alcanzado su punto de inflexión. Lo mismo ocurre en patentes para alimentación animal. Sin embargo, la tecnología de proteína hidrolizada, desde el punto de vista de artículos, entrará a su fase de madurez y declive después del año 2016.
\end{abstract}

Palabras clave: ciclo de vida de la tecnología; curvas en S; probióticos, proteína hidrolizada, alimentación animal

\section{Technology Life Cycle and S Curves applied to Byproducts of the Fish Farming Industry}

\begin{abstract}
The life cycle of available technologies through the S-curves method on three technologies is analyzed: hydrolyzed protein, animal feeding and probiotics, related to products of the fish industry. For this, strategies were constructed to search articles and patents in specialized databases such as Scopus and Free patents online. For the analysis of the information, $\mathrm{S}$ curves were used for calculating the inflection point. This determines the state of technology within its life cycle and helps decision making regarding the right time to exercise mechanisms of intellectual property and technology law, and suggest strategies of monitoring and investment. Among the most important results it is observed that in articles, two of the technologies discussed, hydrolyzed protein and probiotics have not reached their turning point, and the same happened with patent for animal feeding. However, the technology of hydrolyzed protein, from the point of view of the articles, will enter its phase of maturity and decline after 2016.
\end{abstract}

Keywords: technology life cycle; S curves; probiotics, hydrolyzed protein, animal feeding 


\section{INTRODUCCIÓN}

Este artículo comprende la realización de un estudio de curvas en $\mathrm{S}$ en tecnologías para subproductos de la agroindustria piscícola, las herramientas y técnicas utilizadas brindan un elemento de valor agregado para la toma de decisiones relacionadas con el ciclo de vida de la tecnología, es decir, saber si es emergente, entrante, clave, madura 0 en declive, aplicar estrategias de monitoreo/vigilancia, inversión o no sobreinversión, y establecer el momento adecuado para adoptar mecanismos de derecho tecnológico y propiedad intelectual (Pérez, 2001; Ortiz y Pedroza, 2006), esto es importante ya que es conveniente reducir la incertidumbre en toma de decisiones relacionada con el estado en que una tecnología se encuentra (es decir, antes o después de su punto de inflexión) lo que puede llevar a los interesados en la gestión tecnológica a tomar mejores decisiones frente a inversiones en mecanismos de protección (antes del punto de inflexión) o en la no sobreinversión en una tecnología (después del punto de inflexión). El proyecto base del estudio se direccionó en función de tres tecnologías prioritarias que fueron: proteína hidrolizada, probióticos y alimentación animal con énfasis en artículos y patentes, los análisis de los modelos de curvas en $S$ se aplicaron a las series de datos de artículos y patentes acumulados, (Hernández et al, 2016; Zartha et al, 2016) los resultados obtenidos ayudaran como principio para la toma de decisiones estratégicas en el sector piscícola y servirá de base para estudios posteriores en prospectiva tecnológica.

La metodología de curvas en "S" sirve para articular la información de artículos y patentes relacionándola con productos o servicios que se pretendan generar con las tecnologías prioritarias alrededor de los subproductos piscícolas. Los principales objetivos del estudio presentado en el paper, comprenden: (1) realizar la búsqueda de patentes, artículos y documentos técnicos relacionados con tres tecnologías prioritarias en derivados piscícolas; (2) aplicar modelos de curvas en "S" en las tres tecnologías prioritarias seleccionadas; y (3) calcular los puntos de inflexión de las tecnologías prioritarias seleccionadas de derivados piscícolas.

Desde algún tiempo atrás, estudiosos de la tecnología han intentado entender su comportamiento y las estrategias que pueden ser implementadas en cada una de las fases de su trayectoria a través del tiempo y una alternativa para dicho análisis son los modelos de curvas en "S", mediante los cuales es posible identificar el estado de una tecnología: emergente, entrante, clave, madura o en declive; la estrategia a seguir sería: monitoreo, inversión selectiva, sobreinversión (Ortiz \& Pedroza, 2006; Villa, 2015; Mercado, 2013; Arango, 2015) y el comportamiento de la tecnología en cada fase: inicial, crecimiento temprano, crecimiento tardío y madurez (Pérez, 2001; Pérez, 2004) (Figura 1).

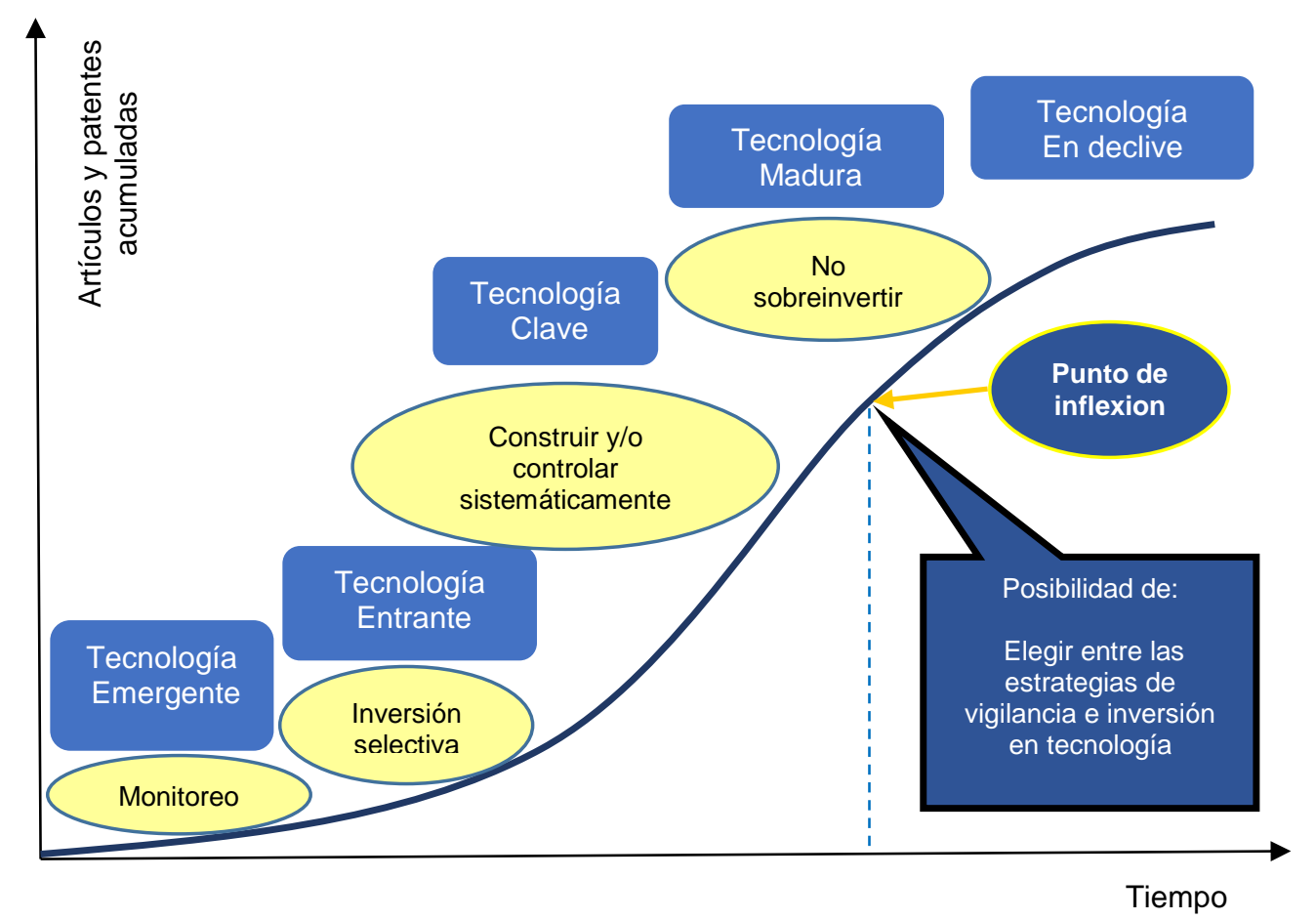

Fig. 1: Estado de la tecnología

El ciclo de vida de la tecnología, la comprensión del fenómeno de la difusión de innovaciones tecnológicas y la adopción de las mismas son otras características medibles a través de las curvas en S (Tidd \& Bessant, 2013; Aguilar et al, 2012; Schilling, 2009). La figura 2 muestra una curva en forma de S en la cual se identifican las etapas de una tecnología. 


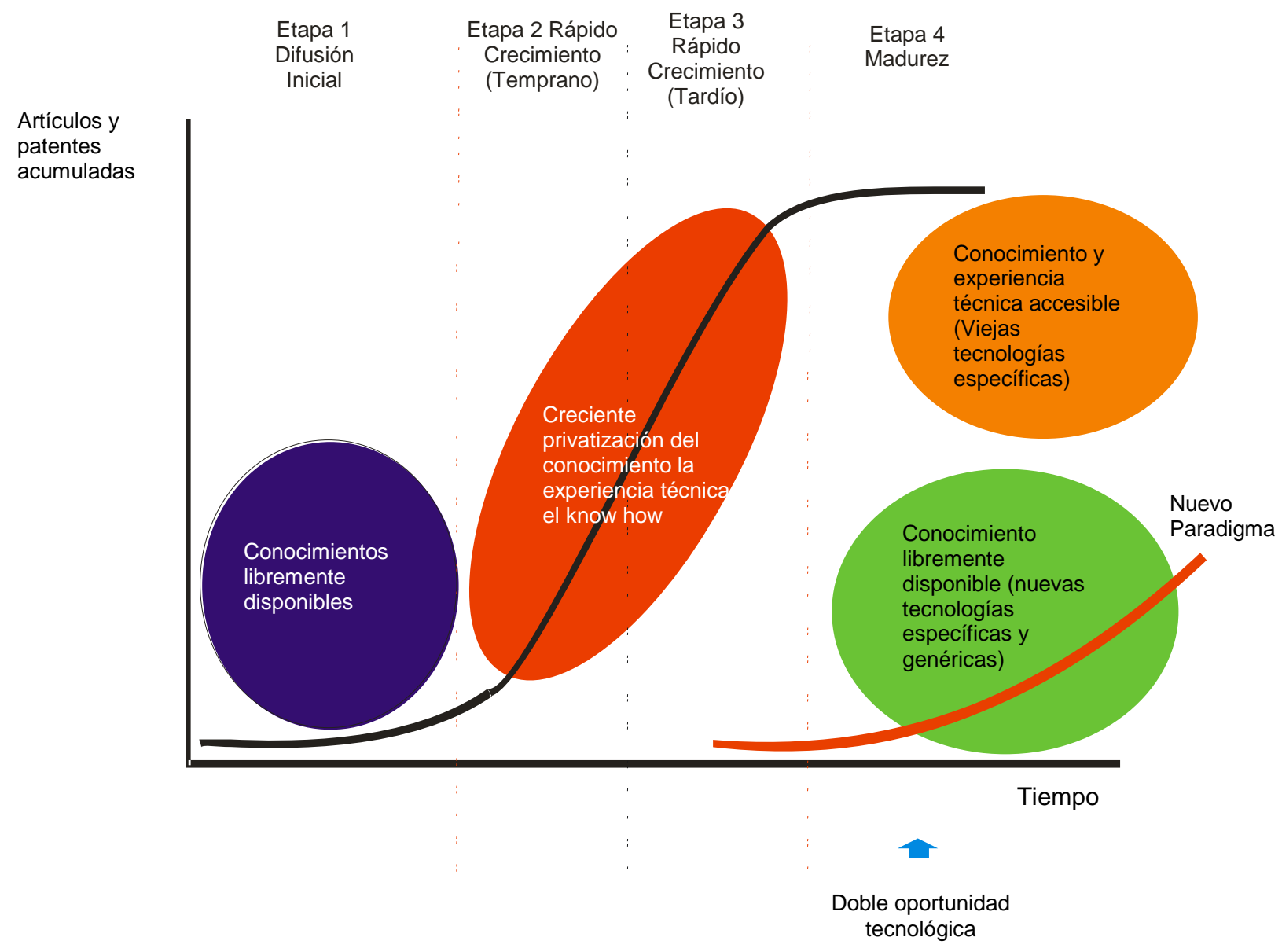

Fig. 2: Fases de una Tecnología

Por otro lado, las innovaciones tienen asociadas una serie de incertidumbres de mercado, de comercio y tecnológicas, las cuales se incrementan con la necesidad de información o conocimiento sobre las variables que intervienen en el proceso de lanzamiento de un nuevo producto o mejora de uno existente (Afuah, 1999; Kotler, 1996; Pérez, 2004; Kucharavy, 2008; por Kucharavy y De Guio, 2009). De igual manera, la incertidumbre tecnológica se refiere a la necesidad de conocimiento adicional sobre los componentes y la forma en que se relacionan los métodos y las técnicas para que el nuevo producto funcione de forma adecuada (Zartha et al, 2014). Según (Zartha et al, 2014) "Estas incertidumbres pueden ser reducidas por medio del estudio de las regularidades tecnológicas, de las regularidades del mercado y del uso de estrategias de innovación adecuadas. La forma más adecuada para analizar las regularidades tecnológicas se realiza por medio de las curvas en S, de esta forma la empresa puede analizar el desempeño de la innovación en el tiempo. De igual manera las regularidades del mercado pueden ser estudiadas por medio de las curvas en S". (p. 2).

El presente artículo contiene la aplicación de una metodología para calcular el punto de inflexión de tres tecnologías relacionadas con innovaciones en subproductos piscícolas, se presentan en un principio los materiales y métodos utilizados haciendo énfasis en la necesidad de alinear procedimientos matemáticos con aplicaciones en curvas en $S$ en ciclo de vida de la tecnología, se mencionan las bases de datos utilizadas estrategias de búsqueda, modelos matemáticos aplicados, procedimiento para calcular el punto de inflexión y las posibles estrategias para la toma de decisiones relacionadas con el estado de las tecnologías analizadas, posteriormente se presentan los resultados y discusión y se finaliza con las conclusiones y referencias.

\section{MATERIALES Y MÉTODOS}

"La metodología comprende cuatro fases las cuales tienen en cuenta trabajos previos en cuanto a curvas en S a nivel cualitativo (Ortiz y Pedroza, 2006; Burgelman, 2009; Schilling, 2013; Tidd y Bessant, 2013), y a nivel cuantitativo (Kucharavy, 2007; Kucharavy, 2009; Schilling, 2009; Foster, 1986), estas cuatro fases resumen los pasos a tener en cuenta para el cálculo del punto de inflexión partiendo de parámetros de desempeño acumulados a través de regresión no lineal."

Fase I: En esta fase de búsqueda de información, se investigaron las bases de datos científicas, tales como Scopus bases de patentes tales como PatentScope y Free Patents on-Line, esta última contiene datos de 
patentes de Japón, Estados Unidos, OMPI - Organización mundial de la propiedad intelectual, Alemania y Europa. Las patentes obtenidas a través de Freepatents on-Line se analizaron para validar su relación con las tres tecnologías derivados piscícolas, Los artículos se analizaron a partir de la base de datos Scopus, y se validó su relación con derivados piscícolas.

Fase II: Los datos obtenidos sobre patentes y artículos se acumularon en el tiempo y se les aplicaron 13 modelos (Sigmoidal 3, Sigmoidal 4, Sigmoidal 5, Logístico 3, Logístico 4, Weibull 4, Weibull 5, Gompertz 3, Gompertz 4, Gompertz 5, Sigmoidal Hill 4, Chapman 3 parameter, Chapman 4 parameter) de regresión no lineal: los modelos se analizaron a través del software SigmaPlot versión gratuita.

Para curvas en $\mathrm{S}$ con base en las series de tiempo obtenidas en la fase inicial, se procedió a la construcción de las curvas en $S$ en artículos y patentes en las tecnologías seleccionadas. Se realizó la búsqueda en bases especializadas como Scopus y bases de datos de patentes (Americana, japonesa, OMPI, entre otras) sobre artículos y patentes relacionados con las tecnologías seleccionadas. Para garantizar la validez y exactitud de los datos se elaboran estrategias de búsqueda, las cuales fueron revisadas por un experto en el tema. Se tabularon los datos obtenidos de artículos y patentes, especificando su respectivo año y cantidad, y se acumularon los valores con el objetivo de introducirlos como parámetros de entrada en el software Sigmaplot y a través de regresiones no lineales se aplicaron 13 modelos, de los cuales se seleccionó el de mejor ajuste y se obtuvieron las respectivas curvas en S. Se aprobaron y validaron las curvas obtenidas teniendo en cuenta los datos estadísticos arrojados por el software: $\mathrm{R}^{2}$ ajustado, valor $\mathrm{T}$, valor $\mathrm{P}$ y Durbin Watson (DW). Posteriormente se analizó el ciclo de vida de las tecnologías seleccionadas teniendo en cuenta los puntos de inflexión obtenidos, en la figura 3 se observa un resumen de la metodología sugerida.

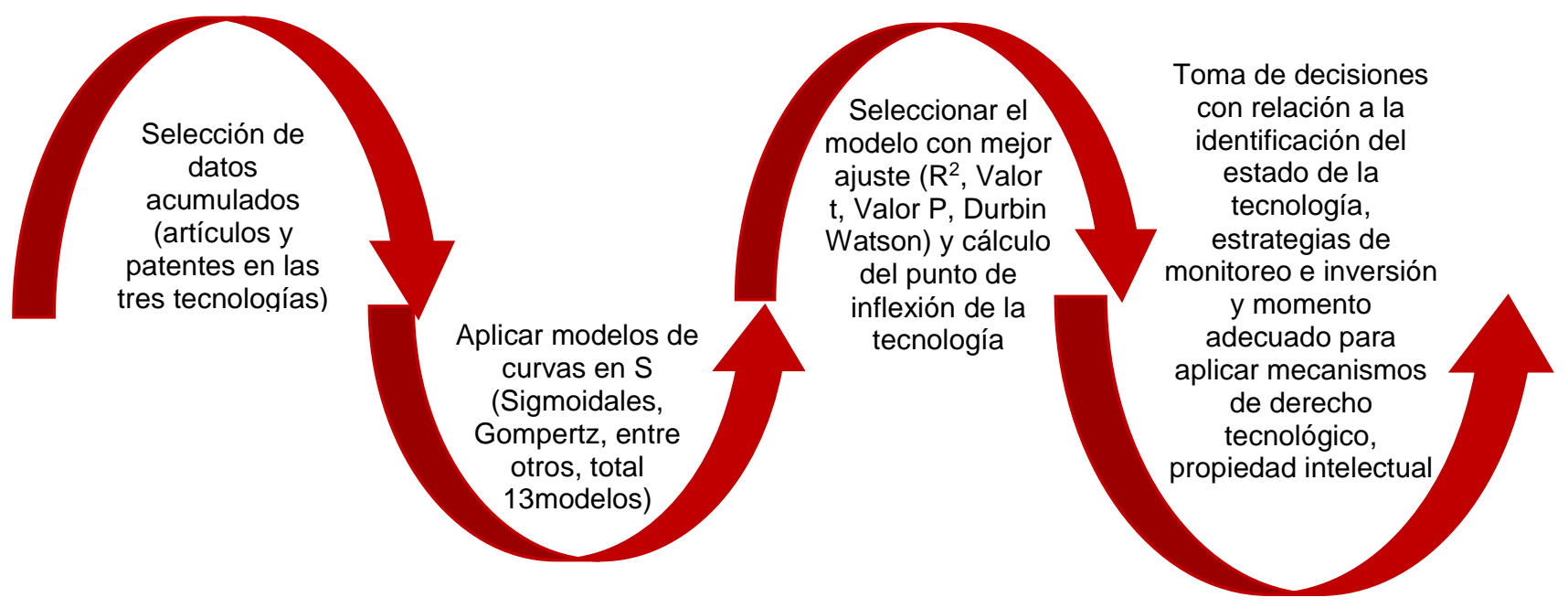

Fig. 3: Metodología para el análisis de una tecnología a través de curvas en S

Fase III: Se calcularon los puntos de inflexión en cada modelo. Estos fueron validados con los valores $\mathrm{R}^{2}$ ajustado, valor $\mathrm{T}$, valor $\mathrm{P}$ y Durbin Watson, se aclara que los parámetros que validan la hipótesis del valor del punto de inflexión son el valor t (que sea mayor que 2 o menor que -2) y el valor $p(q u e ~ s e a<0.005)$.

Fase IV: Con base en los puntos de inflexión obtenidos en cada tecnología se propusieron estrategias para la toma de decisiones relacionadas con el estado de las tecnologías analizadas (emergente, entrante, clave, madura o en declive).

\section{RESULTADOS Y DISCUSIÓN}

Como se mencionó con anterioridad, a nivel cualitativo las curvas en $\mathrm{S}$ han tenido diversas aplicaciones en tecnología e innovación (Schilling, 2013; Burgelman, 2009; Tidd, 2013) y han mostrado utilidad cuando se quieren realizar aproximaciones al ciclo de vida de la tecnología, momento adecuado para generar mecanismos de derecho tecnológico y propiedad intelectual (Pérez, 2001) y posibilidad de aplicar estrategias de monitoreo e inversión (Ortiz y Pedroza, 2006).

Gracias a los desarrollos matemáticos y estadísticos previos sobre la curva en S (llamada también curva sigmoidal o logística) el enfoque cualitativo puede ser complementado con un análisis cuantitativo a través de regresión no lineal y el cálculo y validación del punto de inflexión, en este caso este enfoque mixto puede ser 
de utilidad para reducir la incertidumbre en la toma de decisiones en el sector piscícola, en especial para las nuevas apuestas relacionadas con innovaciones en subproductos piscícolas, donde en el corto y mediano plazo se tomarán decisiones sobre adquisición, absorción, transferencia y uso de tecnologías relacionadas con probióticos, proteína hidrolizada y alimentación animal.

Las tres tecnologías analizadas en esta investigación presentan parámetros de desempeño tales como artículos y patentes que pueden utilizarse para comprender su comportamiento a través del tiempo (aunque si existieran otros parámetros de desempeño tales como eficiencia de la tecnología se podría realizar cálculos complementarios), ahora, a nivel general, una tecnología analizada a través de curvas en $\mathrm{S}$ puede tener los siguientes comportamientos:

Si la tecnología presenta punto de inflexión en una fecha anterior al momento de realizar el análisis, entonces se puede establecer que desde ese momento (o fecha del punto de inflexión) la tecnología entra a la fase de madurez - declive, ya pasó el momento adecuado para ejercer mecanismos de derecho tecnológico y propiedad intelectual y es mejor no sobre invertir en ella. Ahora si la tecnología presenta punto de inflexión en una fecha posterior al momento de realizar el cálculo (es decir, en un momento o fecha futura con relación al momento del cálculo), entonces la tecnología analizada no ha llegado a su fase de madurez - declive, es un momento adecuado para ejercer mecanismos de derecho tecnológico y propiedad intelectual y la estrategia de monitoreo e inversión selectiva sería adecuada.

Las tres tecnologías fueron analizadas a través de curvas en S con dos parámetros de desempeño, patentes y artículos acumulados, con las tecnologías seleccionadas: proteína hidrolizada, alimentación animal y probióticos se construyeron las estrategias de búsqueda (tabla 1) en artículos y patentes para ser utilizadas en bases de datos especializadas, en este caso, dada su pertinencia, fueron seleccionadas: Scopus, para la búsqueda de artículos y Free Patents On-line, para la búsqueda de patentes.

Tabla 1: Estrategias de búsqueda

Estrategias de búsqueda Artículos

Estrategias de búsqueda Patentes
TITLE-ABS-KEY((hydrolyzed protein AND fish) AND NOT (hydrolyzed vegetable protein)) TITLE-ABS-KEY(formulation of concentrates AND fish) TITLE-ABS-KEY(probiotics AND fish products)

ABST((hydrolyzed protein AND fish) AND NOT (hydrolyzed vegetable protein)) ABST(formulation of concentrates AND fish) ABST(probiotics AND fish)

En la tabla 2 se muestran las series de datos de "probióticos" obtenidas con las estrategias de búsqueda de artículos, estas series fueron ingresadas al software Sigmaplot para hallar los puntos de inflexión con base en artículos, el procedimiento fue similar para las tecnologías de alimentación animal y proteína hidrolizada. En la tabla 3 se observa las series de datos de "probióticos" obtenidas con las estrategias de búsqueda de patentes, estas series fueron ingresadas al software Sigmaplot para hallar los puntos de inflexión con base en patentes, el procedimiento fue similar para las tecnologías de alimentación animal y proteína hidrolizada. Después de aplicar los trece modelos de curvas en $\mathrm{S}$ a la serie de datos de artículos en las tres tecnologías, se obtuvieron las siguientes curvas: figura 4, Proteína hidrolizada, figura 5, Probióticos.

En la figura 4, el punto de inflexión con base en artículos corresponde al año 2016, esto fue validado ya que el valor $t$ se encontró fuera del rango entre -2 y +2 y el valor $p$ fue menor que 0.005 . En la figura 5 , el punto de inflexión con base en artículos para probióticos corresponde al semestre 64 a 65, de igual forma, el valor $t$ se encontró fuera del rango entre $-2 \mathrm{y}+2$ y el valor $p$ fue menor que 0.005 .

Tabla 2: Artículos acumulados en la tecnología "Probióticos"

\begin{tabular}{|c|c|c|c|c|c|c|}
\hline \multicolumn{9}{|c|}{ Artículos } \\
\hline $19911(0)$ & $1995-1(1)$ & $1999-1(1)$ & $2003-1(9)$ & $2007-1(29)$ & $2011-1(63)$ & $\begin{array}{c}2015-1 \\
(125)\end{array}$ \\
\hline $1991-2(1)$ & $1995-2(1)$ & $1999-2(1)$ & $2003-2(12)$ & $2007-2(23)$ & $2011-2(72)$ & $\begin{array}{c}2015-2 \\
(136)\end{array}$ \\
\hline $1992-1(1)$ & $1996-1(1)$ & $2000-1(2)$ & $2004-1(14)$ & $2008-1(37)$ & $2012-1(79)$ & $\begin{array}{c}2016-1 \\
(143)\end{array}$ \\
\hline $1992-2(1)$ & $1996-2(1)$ & $2000-2(2)$ & $2004-2(14)$ & $2008-2(39)$ & $2012-2(86)$ & $\begin{array}{c}2016-2 \\
(144)\end{array}$ \\
\hline $1993-1(1)$ & $1997-1(1)$ & $2001-1(3)$ & $2005-1(17)$ & $2009-1(42)$ & $2013-1(96)$ & \\
\hline $1993-2(1)$ & $1997-2(1)$ & $2001-2(3)$ & $2005-2(20)$ & $2009-2(44)$ & $2013-2(102)$ & \\
\hline $1994-1(1)$ & $1998-1(1)$ & $2002-1(5)$ & $2006-1(23)$ & $2010-1(50)$ & $2014-1(115)$ & \\
\hline $1994-2(1)$ & $1998-2(1)$ & $2002-2(7)$ & $2006-2(27)$ & $2010-2(58)$ & $2014-2(117)$ & \\
\hline
\end{tabular}


Tabla 3: Patentes acumuladas en la tecnología "Probióticos"

\begin{tabular}{|l|l|l|l|l|c|}
\hline \multicolumn{7}{|c|}{ Patentes } \\
\hline $1995-1(1)$ & $1999-1(9)$ & $2003-1(13)$ & $2007-1(36)$ & $2011-1(65)$ & $2015-1(125)$ \\
\hline $1995-2(2)$ & $1999-2(9)$ & $2003-2(16)$ & $2007-2(40)$ & $2011-2(73)$ & $2015-2(132)$ \\
\hline $1996-1(3)$ & $2000-1(9)$ & $2004-1(18)$ & $2008-1(42)$ & $2012-1(80)$ & $2016(137)$ \\
\hline $1996-2(7)$ & $2000-2(9)$ & $2004-2(20)$ & $2008-2(48)$ & $2012-2(92)$ & \\
\hline $1997-1(7)$ & $2001-1(9)$ & $2005-1(23)$ & $2009-1(50)$ & $2013-1(92)$ & \\
\hline $1997-2(7)$ & $2001-2(9)$ & $2005-2(25)$ & $2009-2(55)$ & $2013-2(99)$ & \\
\hline $1998-1(9)$ & $2002-1(11)$ & $2006-1(26)$ & $2010-1(58)$ & $2014-1(110)$ & \\
\hline $1998-2(9)$ & $2002-2(11)$ & $2006-2(28)$ & $2010-2(60)$ & $2014-2(120)$ & \\
\hline
\end{tabular}

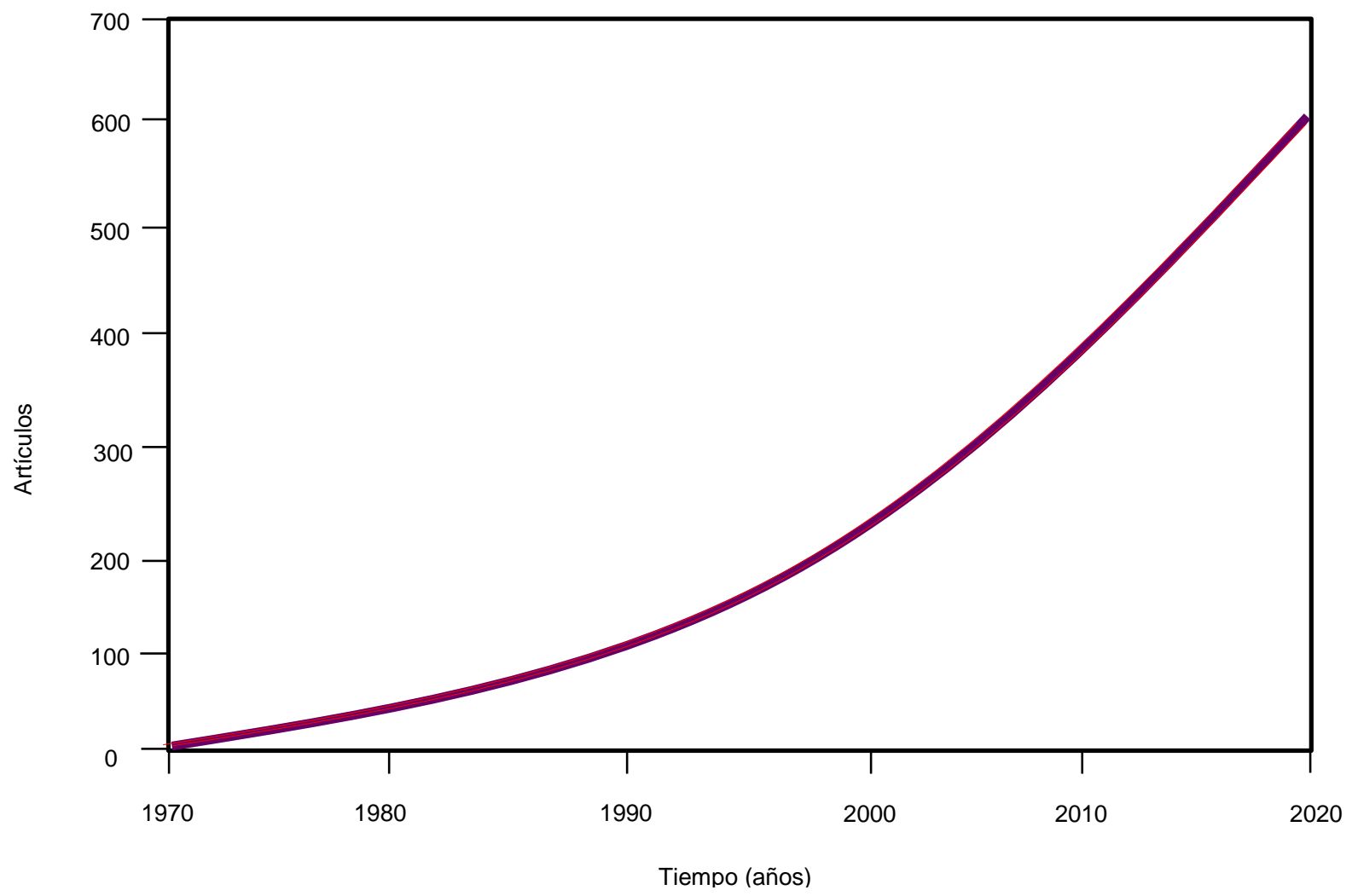

Fig. 4: Curvas obtenidas en artículos en la tecnología proteína hidrolizada

Después de aplicar los trece modelos en la tecnología Alimentación animal, se puede observar que de los trece modelos, ninguno se ajusta a los parámetros estadísticos establecidos. Por ejemplo, al observar el modelos Hill 4, valores $\mathrm{t}(\mathrm{t}(\mathrm{a}), \mathrm{t}(\mathrm{b}), \mathrm{t}(\mathrm{c}), \mathrm{t}(\mathrm{Yo})$ y t) para los que corresponde no se encuentran entre 2 y -2 , y el valor $p$ no es menor que 0.005 , esto valida la hipótesis y no se puede aceptar que ese sea el punto de inflexión. Además el $\mathrm{R}^{2}$ ajustado no es confiable. Esto puede obedecer a que la tecnología se encuentra en una fase anterior al punto de inflexión, es decir es emergente o entrante o clave, desde el punto de vista de artículos no es una tecnología madura o en declive, es decir, se encuentra en la fase exponencial y no se comporta como una curva sigmoidal o curva en $\mathrm{S}$, por lo que ningún modelo puede converger, el año más cercano del posible punto de inflexión es el año 2060, pero esta conclusión solo sería válida si variables externas permanecen constantes a lo largo del tiempo, algo que es improbable en la dinámica actual de innovación y tecnología, otras tecnologías han mostrado comportamiento similar cuando se analizan artículos, tal es el caso de tecnologías en alimentos tales como la ozonización, el secado y la emulsificación (Zartha et al, 2015) y materiales de liberación controlada en biotecnología (Zartha et al, 2016) y en fabricación piloto de guantes biodegradables en las tecnologías de extrusión soplado y coextrusión en almidón termoplástico de yuca. 


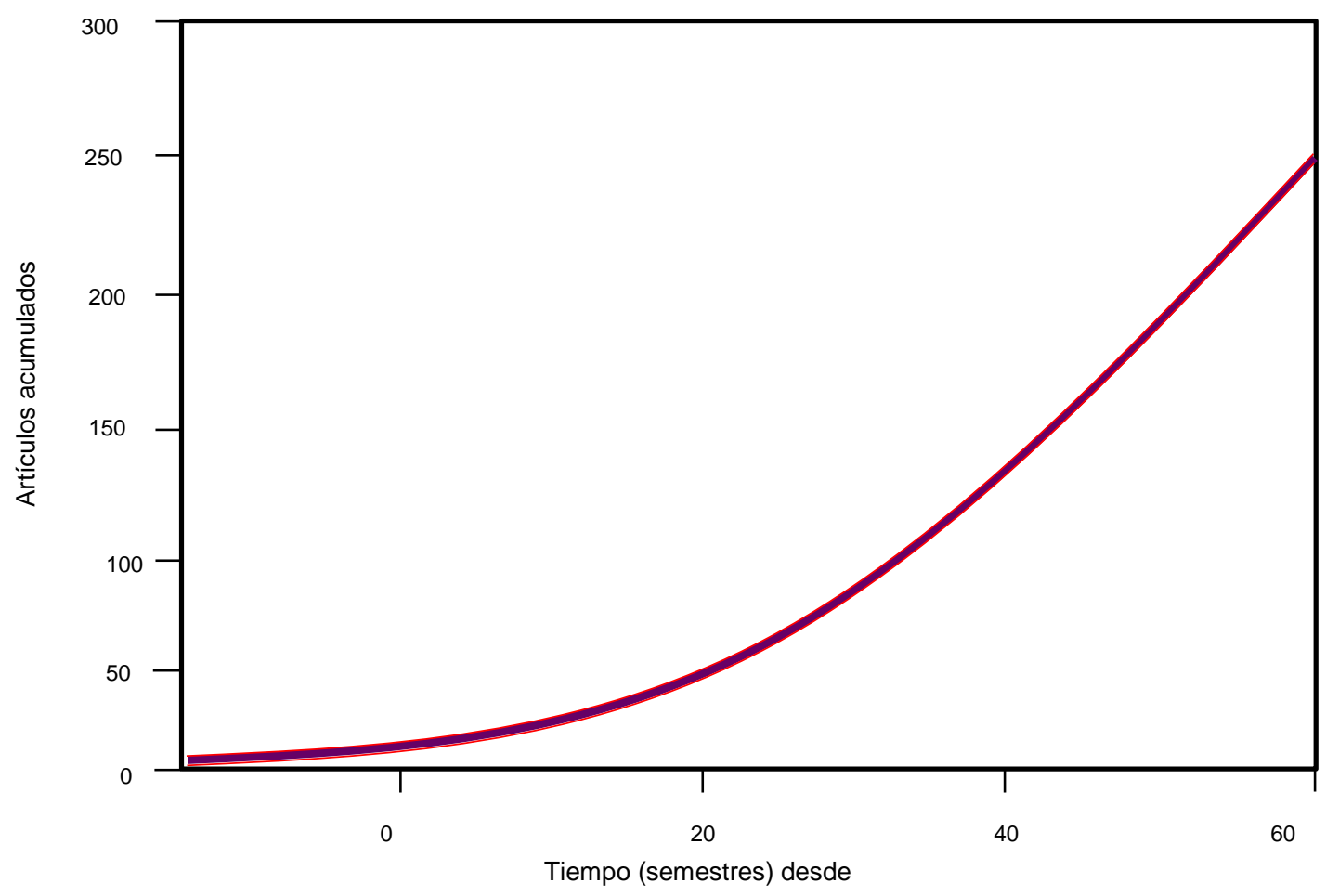

Fig. 5: Curva obtenidas en artículos en la tecnología de Probióticos

Después se aplicaron cada uno de los trece modelos para artículos en la tecnología Proteína hidrolizada, en este caso los modelos sigmoideo 3 y Logístico 3 si convergen y muestran un punto de inflexión de la tecnología en el año 2016, esto se valida ya que los valores $t(t(a), t(b), t(c), t(Y o)$ y $t)$ para los que corresponde son mayores que 2 o menores que -2 , y el valor $p$ es menor que 0.005 . Desde el punto de vista de la curva en $S$, después del año 2016 la tecnología entrará a una nueva fase de madurez y declive, esto desde el punto de vista de artículos, otros estudios de curvas en $\mathrm{S}$ en tecnologías de alimentos en artículos ya habían validado como ganador al modelo sigmoideo, tal es el caso de la emulsificación, sedimentación, centrifugación, molienda, evaporación, plasma frío y altas presiones (Zartha, 2015) y nano lípidos sólidos (Zartha, 2016) y en la tecnología de extrusión reactiva para guantes biodegradables a partir de almidón termoplástico de yuca (Hernández, 2016).

Por último se observan los valores de cada uno de los trece modelos para artículos aplicados a la tecnología probióticos y su validez. Cinco modelos mostraron convergencia, cuatro de ellos evidenciando un punto de inflexión entre 64 a 65 lo cual corresponde a un año superior al 2016, entre 2021 y 2022, lo que sugiere que por ahora la tecnología se encuentra en una fase emergente, entrante o clave y representa un alto potencial para continuar publicando en el tema de probióticos a partir de subproductos piscícolas, de hecho, el primer artículo fue publicado en el año 1991. Después de aplicar los trece modelos de curvas en S a la serie de datos de patentes en las tres tecnologías, se obtuvieron las siguientes curvas: figura 6, Probióticos. En la figura 6 , el punto de inflexión con base en patentes para probióticos corresponde al semestre 43 o año 2016, esto fue validado ya que el valor $t$ se encontró fuera del rango entre $-2 y+2$ y el valor $p$ fue menor que 0.005 .

\section{Patentes en las tres tecnologías}

Después de aplicar los trece modelos en la tecnología Alimentación animal, se puede observar que de los trece modelos, ninguno se ajusta a los parámetros estadísticos establecidos. De igual manera que en los modelos de artículos para esta tecnología puede obedecer a que se encuentra en una fase anterior al punto de inflexión, es decir es emergente o entrante o clave, pero desde el punto de vista de patentes no es una tecnología madura o en declive, es decir, se encuentra en la fase exponencial y no se comporta como una curva Sigmoidal o como curva en S, por lo que ningún modelo puede converger, el año más cercano del posible punto de inflexión es el año 2020, pero esta conclusión solo es válida, como se mencionó anteriormente, si variables externas permanecen constantes, algo que es improbable, por no decir imposible, en la dinámica actual de innovación y tecnología, ahora, situaciones similares se han encontrado en estudios en los últimos años, ya que desde el punto de vista de patentes otras tecnologías han mostrado comportamientos similares como en el caso del estudio de materiales de liberación controlada en biotecnología (Zartha et al, 2016). 


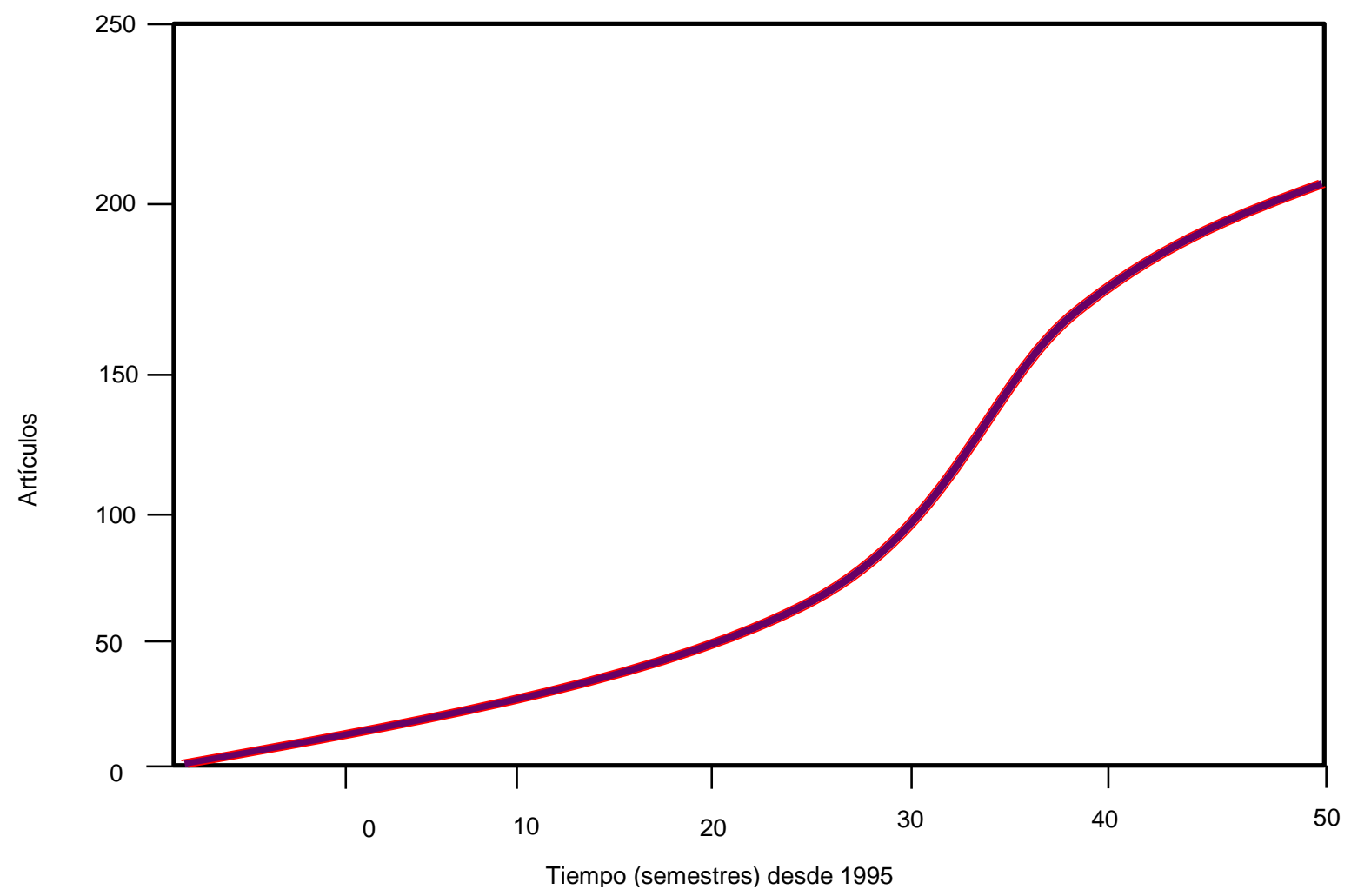

Fig. 6: Curva obtenida en patentes en la tecnología de Probióticos

Después se observan los valores de cada uno de los trece modelos para patentes aplicados a la tecnología Proteína hidrolizada y su validez, ninguno de los modelos se ajusta a los parámetros estadísticos establecidos y no se comporta como una curva Sigmoidal (Curva en S). Aunque ninguno de los trece modelos se ajusta a la serie de datos real, se puede observar que casi todos ellos arrojan valores de punto de inflexión varias décadas por encima del año actual - 2016, dada la incertidumbre que la tecnología representa, no es posible generar conclusiones sobre esas fechas que son muy lejanas al momento del análisis, pero si permite observar, con base en ese parámetro de desempeño de patentes que la tecnología se encuentra en sus primeras fases del ciclo de vida. Por último se observan los valores de cada uno de los trece modelos para patentes aplicados a la tecnología Probióticos y su validez.

En este caso el modelo que cumple con los parámetros de desempeño es el modelo Sigmoidal 3 y tiene su punto de inflexión en el año 2012 lo que quiere decir que la tecnología se encuentra en una fase madura, su convergencia hacia el modelo sigmoidal coincide con otros estudios sobre tecnologías tales como extrusión soplado, co extrusión y extrusión reactiva (Hernández, 2016), las cuales fueron analizadas con los mismos trece modelos del presente estudio.

\section{CONCLUSIONES}

Desde el punto de vista del parámetro de desempeño en patentes, la tecnología de alimentación animal basada en subproductos piscícolas se encuentra en fase anterior a su punto de inflexión, esto quiere decir que las tasas de patentamiento aún son altas y puede ser una buena alternativa pensar en patentar en este momento en este tipo de tecnología. La tecnología de probióticos basados en subproductos piscícolas, se encuentra también en una fase de grandes oportunidades para su publicación, ya que aún no ha llegado a su punto de inflexión.

Desde el punto de vista del parámetro de desempeño en patentes, la tecnología de alimentación animal basada en subproductos piscícolas se encuentra en fase anterior a su punto de inflexión, esto quiere decir las tasas de patentamiento aún son altas y puede ser una buena alternativa pensar en patentar en este momento en este tipo de tecnología. Para la tecnología de Probióticos en patentes es importante resaltar que, desde el punto de vista de ese parámetro, debe estudiarse la estrategia de no sobre invertir en mecanismos de derecho tecnológico y propiedad intelectual en ésta tecnología pero que si es un momento apropiado para su divulgación a través de publicaciones. La tecnología de proteína hidrolizada, desde el punto de vista de artículos, entrará a su fase de madurez y declive después del año 2016. 
De los trece modelos analizados, el que más se ajustó a las tres tecnologías es el modelo sigmoideo, esto coincide con otros estudios sobre tecnologías agroindustriales y no agroindustriales, este se constituye en un buen modelo para realizar nuevas investigaciones sobre ciclo de vida en otras tecnologías agroindustriales.

Es importante pensar en una clasificación de las tecnologías utilizando un indicador mixto de los parámetros de desempeño utilizados, es decir de patentes y artículos, o una forma de graficar y analizar los datos con dos o incluso tres parámetros de desempeño, esto con el fin de generar conclusiones más precisas sobre el ciclo de vida de una tecnología. Puede ser conveniente complementar los cálculos realizados en patentes con una nueva estrategia de búsqueda que contenga las CLAIM o reivindicaciones, ya que allí podrían encontrarse otros documentos diferentes a los arrojados con la estrategia de búsqueda con los abstract.

\section{AGRADECIMIENTOS}

Los autores expresan sus agradecimientos al Departamento Nacional de planeación, al sistema general de regalías de Colombia, la Gobernación del Cauca, al Centro Regional de Productividad e Innovación del Cauca -CREPIC y al grupo de investigación ASUBAGROIN a través del proyecto "Alternativas para el uso de subproductos derivados de la Agroindustria piscícola" ALTPEZ.

\section{REFERENCIAS}

Afuah, A., La dinámica de la innovación organizacional el nuevo concepto para lograr ventajas competitivas y rentabilidad, Universidad de Oxford (1999)

Aguilar, S., Ávalos, A., Giraldo, D., Quintero, S., Zartha, J., Cortés, F., Las curvas en S como herramienta para la medición de los ciclos de vida de productos, Journal of Technology Management \& Innovation, ISSN: 07182724, 7(1) (2012)

Arango, J., Duque, H. Análisis de la difusión de automóviles particulares en diferentes ciudades de Colombia por medio de curvas en S. Tesis de Magister. Escuela de Ingenieria Agroindustrial. Universidad Pontificia Bolivariana, Medellín, Colombia (2015)

Burgelman, R., Christensen, C., Wheelwright, S., Strategic Management of technology and innovation, $5^{a}$ Edición, McGraw-Hill, Boston (2009)

Foster, R., Working The S-Curve: Assessing Technological Threats, Research Management, 29(4), (1986)

Hernández, R., Villada H. y otros 10 autores, Vigilancia tecnológica y análisis del ciclo de vida de la tecnología: evaluación del potencial comercial de un prototipo de guantes biodegradables a partir de almidón termoplástico de yuca, Revista Espacios, ISSN: 0798-1015, 37(13), 27 - 28 (2016)

Kotler, P., Dirección de mercadotecnia, análisis, planeación, implementación y control, $8^{a}$ edición, Pretince Hall (1996)

Kucharavy, D., Guio, R., Application of S-shaped curve, Procedia Engineering (2007)

Kucharavy, D., De Guio, R., Logistic Substitution Model and Technological Forecasting, Journal Part of the Real Innovation, (2009)

Mercado, H. Las curvas en "S" como herramienta para evaluar el desempeño de las innovaciones en la formación del programa jóvenes rurales emprendedores - Sena. Tesis de especialización. Universidad Pontificia Bolivariana, Medellín, Colombia (2013)

Ortiz, S., Pedroza, A., ¿Qué es la gestión de la innovación y la tecnología? Journal of Technology Management and Innovation, ISSN: 0718-2724, 1(2), 64-82 (2006)

Pérez, C., El cambio tecnológico y las oportunidades de desarrollo como blanco móvil, Revista de la CEPAL, ISSN: 0252-0257, (75), 115-136 (2001)

Pérez, C., Technological revolutions, paradigm shifts and socio-institucional change, Globalization, Economic Development and Inequality, Edward Elgan Publishing limited, Massachusetts, Estados Unidos, 217 - 220 (2004)

Porter, M., La ventaja competitiva de las naciones. Ser competitivos, Editorial Desuto, Boston Estados Unidos (1999)

Shilling, M., Strategic management of technological innovation, 4ª edición, McGraw-Hill, New York (2013) 
Schilling, M., Esmundo, M., Technology S-curves in renewable energy alternatives: Analysis and implications for industry and government, Energy Policy, 37(5,) 1767-1781 (2009)

Tidd, J., Bessant, J., Managing Innovation. Integrating Technological Market and Organizational Change, $5^{\underline{a}}$ edición, Reino Unido (2013)

Villa, E. Análisis de la Gestión de Tecnologías Emergentes (GTE) en grupo de investigación colombiano e identificación de brechas respecto a referentes internacionales. Tesis de Magister. Departamento de Ingeniería de la Organización. Universidad Nacional, Medellín, Colombia (2015)

White, M., Bruton, G., A Strategic Approach. The Management of technology and Innovation. Mason, OH. Thompson South-Western (2007)

Zartha, J., Arango, B., Hernández, Z., Moreno, J, Análisis del ciclo de vida la tecnología a través de curvas en S: Aplicación en operaciones unitarias en alimentos, Revista Espacios, ISSN: 0798-1015, 35 (7), 1 - 2 (2014)

Zartha, J., Arango, B., Hernández, R., Gamaliel, J., Orozco, G., Curvas en S y análisis de clúster en ciclo de vida de la tecnología: Aplicación en 11 tecnologías en alimentos, Revista Espacios, ISSN: 0798-1015, 36 (12), $5-6(2015)$

Zartha, J., Palop, F., Velez. Avalos, A. S-Curve analysis and technology life cycle. Application in series of data of articles and patents, Revista Espacios, ISSN: 0798-1015 37(7), 19 - 20 (2016) 